\title{
Professional responsibilities regarding the provision, publication, and dissemination of patient phenotypes in the context of clinical genetic and genomic testing: points to consider-a statement of the American College of Medical Genetics and Genomics (ACMG)
}

\author{
Lynn W. Bush, PhD, MS ${ }^{1}$, Anita E. Beck, MD, PhD², Leslie G. Biesecker, MD³, James P. Evans, MD, PhD ${ }^{4}$, \\ Ada Hamosh, $\mathrm{MD}^{5}$, Ingrid A. Holm, MD, MPH${ }^{6}$, Christa L. Martin, PhD ${ }^{7}$, C. Sue Richards, $\mathrm{PhD}^{8}$ and \\ Heidi L. Rehm, PhD $^{9}$; on behalf of the ACMG SELI committee
}

Disclaimer: This Points to Consider document is designed as an educational resource to provide best practices for medical genetic clinicians, laboratories, and journals regarding the provision, publication, and dissemination of patient phenotypes in the context of genomic testing, clinical genetic practice, and research. While the goal of the document is the improvement of patient care, the considerations and practices described should not be considered inclusive of all proper considerations and practices or exclusive of others that are reasonably directed to obtaining the same goal. In determining the value of any practice, clinicians, laboratories, and journals should apply their own professional standards and judgment to the specific circumstances presented. The content of this article is solely the responsibility of the authors and does not necessarily represent the official views of the authors' affiliated institutions.

Genet Med advance online publication 11 January 2018

Key Words: Genomics; Guidelines; Phenotypes; Publications; Sequencing
As comprehensive genetic and genomic testing are increasingly incorporated into the care of patients, there is a critical need for heightened consideration of professional responsibilities and implications in the provision and publication of patient phenotypic information. We offer a best-practice perspective focused on the best interest of the patient and recognition of the roles of laboratories, clinicians, and journals toward this endeavor. This Points to Consider document expands on the American College of Medical Genetics and Genomics' recent position statements on genomic data sharing ${ }^{1}$ and standards for the interpretation of sequence variants. ${ }^{2}$ Each document emphasizes how vital it is for the richest possible clinical and genetic data to be made available in the clinical care process and wider medical and scientific communities to facilitate broad success in reaching a clinical diagnosis, best-possible variant interpretation, and wellcurated literature and databases. The following framework represents key best-practice responsibilities applied to professionals involved in the provision and publication of phenotypic and genotypic information grounded on the ethical construct of beneficence and the premise that ensuring optimal data sharing is the gold standard among clinicians, diagnostic laboratories, and researchers.

\section{PROVISION OF CLINICAL DATA DURING TESTING}

The current model of genetic and genomic testing assumes the laboratory has access to clinical data sufficient to prioritize variants for analysis and accurately interpret variants during clinical reporting. Certain types of conditions and phenotypes that prompt genetic testing are amenable to formulaic reporting on requisition forms. For example, in suspected cancer predisposition syndromes it is simple to provide types of cancer in an individual and their relatives. However, in other situations (e.g., syndromic presentations) phenotypes can be quite complex and varied, making it more difficult to communicate pertinent phenotypic data. In these areas, where "open text" fields on a requisition form may be necessary for

\footnotetext{
${ }^{1}$ Department of Pediatrics, Division of Clinical Genetics, Columbia University Medical Center, New York, New York, USA; ${ }^{2}$ Department of Pediatrics, Division of Genetic Medicine, University of Washington and Seattle Children's Hospital, Seattle, Washington, USA; ${ }^{3}$ Medical Genomics and Metabolic Genetics Branch, National Human Genome Research Institute, National Institutes of Health, Bethesda, Maryland, USA; ${ }^{4}$ Department of Genetics, University of North Carolina at Chapel Hill, Chapel Hill, North Carolina, USA; ${ }^{5}$ McKusick-Nathans Institute of Genetic Medicine, Johns Hopkins University, Baltimore, Maryland, USA; ${ }^{6}$ Division of Genetics and Genomics, Boston Children's Hospital and Department of Pediatrics, Harvard Medical School, Boston, Massachusetts, USA; ${ }^{7}$ Autism and Developmental Medicine Institute, Geisinger Health System, Danville, Pennsylvania, USA; ${ }^{8}$ Knight Diagnostic Laboratories, Oregon Health and Science University, Portland, Oregon, USA; ${ }^{9}$ Department of Pathology, Brigham \& Women's Hospital and Harvard Medical School, Boston, Massachusetts, USA. Correspondence: Lynn W. Bush (lwb25@cumc.columbia.edu)

The Board of Directors of the American College of Medical Genetics and Genomics approved this statement on 29 August 2017.
} 
communication of phenotypic features to the laboratory, it will be particularly important, yet potentially challenging, for clinicians to take adequate time and thought to include the most pertinent phenotypic findings. We recognize this ideal may be difficult to realize given limited resources, including time and staff, and that viable alternatives in the future may involve structured phenotype entry into the electronic health record with single-push forwarding to laboratories as well as less clinical correlation by laboratories and more by ordering clinicians.

\section{The following are best practices:}

- Clinicians should provide detailed phenotypic and family history data to clinical laboratories, including phenotypic features the clinician may not initially see as relevant, appreciating the potential for laboratories to find unsuspected etiologies or multiple causes for independent clinical findings. Attaching the clinic note and family history table or pedigree (stripped of nonproband protected health information) may be considered sufficient for this purpose. If the ordering clinician receives a request from the laboratory to provide additional information (subsequent bullet), reasonable effort should be made to provide the requested information.

- Clinical laboratories should ensure that clinical information is considered in the interpretation of genetic and genomic findings related to the test indication. Laboratories should request these data up front, but if insufficient clinical information is supplied to adequately interpret findings, additional information necessary to interpret the genetic data should be requested.

- The clinician and laboratory should try to discuss and identify further studies that may improve the interpretation, potentially as an iterative exchange in response to genotypic information generated by clinical laboratories, with the clinician sharing multiple rounds of information possibly including physical examination, laboratory studies, imaging, and family history, when feasible. However, additional studies should not delay a laboratory report.

- Given the utility of segregation, de novo, and allelic (cis/ trans configurations) evidence in the evaluation of variant pathogenicity, informative family member samples should also be requested when needed to clarify variants of uncertain significance that have a high prior probability of being causative for the indication for testing. If family member samples are sent, genotype-phenotype correlation should be performed to ensure consistency with proband variant interpretation.

- Clinical laboratories should also provide clinicians with access to knowledgeable staff members of the laboratory (genetic counselors, laboratory directors, etc.) capable of discussing results, variant evidence, genotype-phenotype correlations, and how the provision of phenotypic data will inform the interpretation of variants.

\section{AUTHORSHIP ATTRIBUTION AND PUBLICATION RESPONSIBILITY}

To improve our understanding of genetic conditions and enhance patient care, clinicians and clinical laboratories are encouraged to publish case reports, case series, and aggregate results of genetic testing and to develop publications jointly when both contribute sufficiently to the publication. Criteria to determine authorship should follow established guidelines such as the International Committee of Medical Journal Editors (ICMJE) ${ }^{3}$ with attribution fairly reflecting contribution. Authorship planning should be undertaken early in manuscript preparation, such that clinicians who publish case reports or series should discuss authorship with labs, and labs that publish novel molecular discoveries/phenotypic expansions should discuss authorship with clinicians.

While basic professional rules of conduct for publication are widely promulgated, this Points to Consider document provides more explicit best practices for authorship inclusion, especially to minimize any potential hesitation by clinicians in the provision of phenotypic details necessary for optimal interpretation by clinical laboratories.

Ideally, if the publication is led by one professional discipline, authorship should be considered based on the following points, subject to further contextual nuances:

- Clinicians should include relevant clinical laboratory members as authors on publications if the test results were a critical contribution to the manuscript. When the laboratory data are a major contribution to a clinical paper or if atypical or customized analyses were performed, additional laboratory coauthors should be considered for inclusion in a clinical report. When the clinical laboratory data represents a relatively modest contribution to a detailed clinical report, it may be reasonable to limit laboratory authorship to a single individual. If results are not individually critical, then at least the name of the laboratory (or laboratories) providing testing should be included in the methods section.

- Clinical laboratories should include the contributing clinician(s) on the authorship list if individual-level clinical data are being published (main text or supplementary material) on an individual case or small case series. If patient clinical data are complex and central to a laboratory report, it may be appropriate to consider more than a single clinical coauthor.

- There may be situations that arise when both clinical laboratories and clinicians wish to independently publish findings. In these cases, the two parties need to have a 
dialogue on how to separately focus the papers and allow appropriate recognition of both parties. Moreover, laboratory publication of large aggregate results from genetic testing, such as reporting overall clinical sensitivities and lists of interpreted variants, does not require clinician authorship unless new clinical phenotypes are reported.

- Journals are encouraged, through publication policies, to foster attribution to those who generate and contribute key individual-level clinical data, key genotypic data, or make significant contribution to analysis, interpretation, and provision of phenotypic or genotypic information. Authorship by laboratories or clinicians must be reasonable in number, scaled to the role of the authors in proportion to contribution, and all authors must warrant authorship by contributing sufficiently per ICMJE guidelines. $^{3}$

- Journals should also require that when descriptive patient-level phenotypic data are used in a publication and the phenotypic and genotypic information were obtained through clinical testing, clinicians and clinical laboratories have a responsibility to be transparent regarding potential risks (such as identification and privacy concerns) and benefits of inclusion in the publication. Appropriate consent must be obtained from the families involved; see guidance from ICMJE. ${ }^{4}$

Ideally, the best-practice points discussed in this document should serve as an opportunity to consider ways for improving patient care, our first and foremost goal. Maximizing the provision, publication and dissemination of phenotypic, genotypic, and other clinical information across disciplines will ultimately benefit patients.

\section{ACKNOWLEDGMENTS}

H.L.R. and C.L.M. were supported in part by National Institutes of Health HG006834.

\section{NOTES REGARDING ICMJE GUIDELINES}

3Excerpt: "The ICMJE [International Committee of Medical Journal Editors] recommends that authorship be based on the following 4 criteria: Substantial contributions to the conception or design of the work; or the acquisition, analysis, or interpretation of data for the work; AND Drafting the work or revising it critically for important intellectual content; AND Final approval of the version to be published; AND Agreement to be accountable for all aspects of the work in ensuring that questions related to the accuracy or integrity of any part of the work are appropriately investigated and resolved)."

${ }^{4}$ Clinical information on a patient is considered research by the ICMJE - even if not subject to institutional review board reviewonce published or publicly disseminated. Excerpt: "Patients have a right to privacy that should not be violated without informed consent.... Informed consent should be obtained if there is any doubt that anonymity can be maintained."

\section{DISCLOSURE}

All workgroup members are clinical service providers. The following workgroup members provide laboratory attestations: L.G.B. oversees a CLIA-certified lab at the National Institutes of Health and is an uncompensated consultant to the Illumina Corporation (which runs a clinical lab) as a part of his official duties at the National Institutes of Health; H.L.R. is employed by Partners Healthcare Laboratory for Molecular Medicine and is on the Scientific Advisory Board of Genome Medical; C.S.R. is medical director of Oregon Health \& Science University Knight Diagnostic Laboratories, an academic lab providing clinical genetic testing. The other authors declare no conflict of interest.

\section{REFERENCES}

1. ACMG Board of Directors. Laboratory and clinical genomic data sharing is crucial to improving genetic health care: a position statement of the American College of Medical Genetics and Genomics. Genet Med 2017;19:721-722.

2. Richards S, Aziz N, Bale S, et al. Standards and guidelines for the interpretation of sequence variants: a joint consensus recommendation of the American College of Medical Genetics and Genomics and the Association for Molecular Pathology. Genet Med. 2015;17:405-23.

3. International Committee of Medical Journal Editors. Defining the Role of Authors and Contributors. 2017. http://www.icmje.org/recommendations/ browse/roles-and-responsibilities/defining-the-role-of-authors-and-contributors. html. Accessed 9 February 2017.

4. International Committee of Medical Journal Editors. Protection of Research Participants. 2017. http://www.icmje.org/recommendations/ browse/roles-and-responsibilities/protection-of-research-participants. html. Accessed 9 February 2017. 